\title{
ON KENMOTSU MANIFOLDS SATISFYING CERTAIN CURVATURE CONDITIONS
}

\section{Riddhi Jung Shah}

Journal of Institute of Science and Technology

Volume 22, Issue 2, January 2018

ISSN: 2469-9062 (print), 2467-9240 (e)

\section{Editors:}

Prof. Dr. Kumar Sapkota

Prof. Dr. Armila Rajbhandari

Assoc. Prof. Dr. Gopi Chandra Kaphle

Mrs. Reshma Tuladhar

JIST, 22 (2): 56-62 (2018)

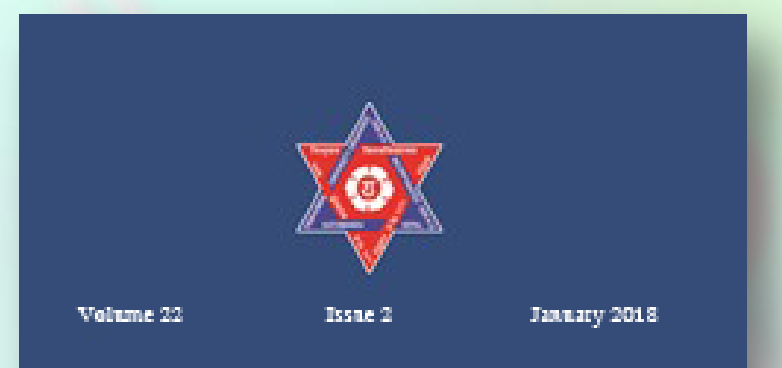

JOURNAL OF INSTITUTE OF SCIENCE AND TECHNOLOGY

Published by:

Institute of Science and Technology

Tribhuvan University

Kirtipur, Kathmandu, Nepal 


\title{
ON KENMOTSU MANIFOLDS SATISFYING CERTAIN CURVATURE CONDITIONS
}

\author{
Riddhi Jung Shah \\ Department of Mathematics, Janata Campus, Nepal Sanskrit University, Dang, Nepal \\ Corresponding E-mail: shahrjgeo@gmail.com
}

Received: 2 February, 2017; Revised: 4 September, 2017; Accepted: 7 September, 2017

\begin{abstract}
The purpose of the present paper is to study certain curvature conditions on Kenmotsu manifolds. It was proved that Kenmotsu manifolds satisfying curvature conditions $R(\xi, X) \cdot B=0, \tilde{C}(\xi, X) \cdot B=0$ and $S(X, \xi) \cdot B=0$ are D-conformally flat. It was also proved that Kenmotsu manifolds satisfying the curvature conditions $P(\xi, X) \cdot B=0, C(\xi, X) \cdot B=0$ and $g(B(\varphi X, \varphi Y) \varphi Z, \varphi W)=0$ are Einstein manifolds with scalar curvature $r=-n(n-1)$. Finally, we gave an example of 3-dimensional Kenmotsu manifold.
\end{abstract}

Keywords: Kenmotsu manifold, D-conformal tensor, Einstein manifold, $\eta$-Einstein, Ricci tensor.

\section{INTRODUCTION}

Kenmotsu studied a class of almost contact Riemannian manifolds (Kenmotsu, 1972). A Kenmotsu manifold is normal but not Sasakian. Moreover, it is also not compact since $\operatorname{div} \xi=n-1$. Kenmotsu showed that locally a Kenmotsu manifold is a warped product $I \times_{f} N$ of an interval $I$ and a Kaehler manifold $N$ with warping function $f(t)=s e^{t}$, where $s$ is a nonzero constant. He also proved that if Kenmotsu manifold satisfies the condition $R(X, Y) \cdot R=0$, then the manifold is of negative curvature -1 . Later, Kenmotsu manifolds have been studied by De and Pathak (2004), Jun et al. (2005), De (2008), De et al. (2009).

In preliminaries we studied some basic relations of Kenmotsu manifolds and D-conformal curvature tensor. We investigated some results on Kenmotsu manifolds satisfying curvature conditions such as

$$
\begin{aligned}
& R(\xi, X) \cdot B=0, P(\xi, X) \cdot B=0, \tilde{C}(\xi, X) \cdot B=0, \\
& C(\xi, X) \cdot B=0, S(X, \xi) \cdot B=0 \text { and } \\
& g(B(\varphi X, \varphi Y) \varphi Z, \varphi W)=0 .
\end{aligned}
$$

Finally, we studied an example of 3-dimensional Kenmotsu manifold.

\section{PRELIMINARIES}

Let $M$ be $\mathrm{n}(\mathrm{n}=2 \mathrm{~m}+1)$-dimensional almost contact manifold equipped with an almost contact metric structure $(\varphi, \xi, \eta, g)$ consisting of a $(1,1)$ tensor field $\varphi$, a contravariant vector field $\xi$, a 1 -form $\eta$ and a compatible Riemannian metric $g$ satisfying

$$
\begin{aligned}
& \left\{\begin{array}{l}
\varphi^{2}(X)=-X+\eta(X) \xi, \eta(\xi)=1, \\
\varphi \xi=0, \eta(\varphi X)=0,
\end{array}\right. \\
& g(X, Y)=g(\varphi X, \varphi Y)+\eta(X) \eta(Y), \\
& g(X, \varphi Y)=-g(\varphi X, Y), \eta(X)=g(X, \xi),
\end{aligned}
$$

for all $X, Y \in \chi(M)$ (Blair, $1976 \&$ 2002). An almost contact metric manifold $M$ is called a Kenmotsu manifold if it satisfies

$$
\begin{aligned}
& \left(\nabla_{X} \varphi\right)(Y)=g(\varphi X, Y) \xi-\eta(Y) \varphi X, \\
& \nabla_{X} \xi=X-\eta(X) \xi,
\end{aligned}
$$

where $\nabla$ denotes the Riemannian connection of $g$ (Kenmotsu, 1972).

In an $n(n=2 m+1)$-dimensional Kenmotsu manifold the following relations hold: 


$$
\begin{aligned}
& \begin{aligned}
\left(\nabla_{X} \eta\right)(Y) & =g(X, Y)-\eta(X) \eta(Y) \\
& =g(\varphi X, \varphi Y),
\end{aligned} \\
& \begin{aligned}
\eta(R(X, Y) Z)=g(X, Z) \eta(Y)-g(Y, Z) \eta(X), \\
R(X, Y) \xi=\eta(X) Y-\eta(Y) X,
\end{aligned} \\
& R(\xi, X) Y=\eta(Y) X-g(X, Y) \xi, \\
& S(X, \xi)=-(n-1) \eta(X), \\
& Q \xi=-(n-1) \xi, \\
& S(\varphi X, \varphi Y)=S(X, Y)+(n-1) \eta(X) \eta(Y),
\end{aligned}
$$

for any vector fields $X, Y, Z \in \chi(M)$, where $R, S$ and $Q$ are the Riemannian curvature, the Ricci tensor and the Ricci operator respectively (Kenmotsu, 1972).

The D-conformal curvature tensor in an $n(n=2 m+1)$-dimensional Riemannian manifold, $n>4$, is defined by

$$
\begin{aligned}
& B(X, Y) Z \\
& =R(X, Y) Z+\frac{1}{n-3}[S(X, Z) Y \\
& -S(Y, Z) X+g(X, Z) Q Y \\
& -g(Y, Z) Q X-S(X, Z) \eta(Y) \xi \\
& +S(Y, Z) \eta(X) \xi-\eta(X) \eta(Z) Q Y \\
& +\eta(Y) \eta(Z) Q X]-\frac{K-2}{n-3}[g(X, Z) Y \\
& -g(Y, Z) X]+\frac{K}{n-3}[g(X, Z) \eta(Y) \xi \\
& -g(Y, Z) \eta(X) \xi+\eta(X) \eta(Z) Y \\
& -\eta(Y) \eta(Z) X],
\end{aligned}
$$

where $K=\frac{2(n-1)+r}{n-2}$ (Chuman, 1983). From (13), we also have

$$
\begin{aligned}
& B(X, Y) \xi=B(\xi, Y) Z=B(X, \xi) Z=0, \\
& \eta(B(X, Y) Z)=0 .
\end{aligned}
$$

Definition: A Kenmotsu manifold $M^{n}$ is said to be $\eta$-Einstein if its Ricci tensor $S$ of type $(0,2)$ is of the form

$$
S(X, Y)=a g(X, Y)+b \eta(X) \eta(Y),
$$

for any vector fields $X$ and $Y$, where $a, b$ are smooth functions on $M$. If $b=0$, then the manifold is said to be an Einstein manifold.

\section{RESULTS AND DISCUSSION}

We proved the following theorems:

Theorem 1. Let $M$ be an $n$-dimensional Kenmotsu manifold satisfying the condition $R(\xi, X) \cdot B=0$. Then the manifold $M$ is D-conformally flat.

Proof. Let us consider an $n$-dimensional Kenmotsu manifold $M$ which satisfies the condition $(R(\xi, X) \cdot B)(U, V) Z=0$. Then, by definition we have

$$
\begin{aligned}
0= & R(\xi, X) B(U, V) Z-B(R(\xi, X) U, V) Z \\
& -B(U, R(\xi, X) V) Z-B(U, V) R(\xi, X) Z .
\end{aligned}
$$

Using (9) in (17) we get

$$
\begin{aligned}
& \eta(B(U, V) Z) X-g(X, B(U, V) Z) \xi \\
& -\eta(U) B(X, V) Z+g(X, U) B(\xi, V) Z \\
& -\eta(V) B(U, X) Z+g(X, V) B(U, \xi) Z \\
& -\eta(Z) B(U, V) X+g(X, Z) B(U, V) \xi=0 .
\end{aligned}
$$

By virtue of (14), (15) and (18) we have

$$
\begin{aligned}
0= & g(X, B(U, V) Z) \xi+\eta(U) B(X, V) Z \\
& +\eta(V) B(U, X) Z+\eta(Z) B(U, V) X .
\end{aligned}
$$

Taking inner product on both sides of (19) by $\xi$ and using (1) and (15) we get

$$
g(X, B(U, V) Z)=0 .
$$

This implies that

$$
B(U, V) Z=0 .
$$

Thus the manifold is D-conformally flat. This completes the proof of the theorem.

Theorem 2. If a Kenmotsu manifold $M^{n}$ satisfies the condition $P(\xi, X) \cdot B=0$, then the manifold is Einstein and the scalar curvature is $r=-n(n-1)$.

Proof. Let $M$ be an $n$-dimensional Kenmotsu manifold. The Weyl projective curvature tensor $P$ of type $(1,3)$ on a Riemannian manifold $(M, g)$ of dimension $n$ is defined by 


$$
\begin{aligned}
& P(X, Y) Z \\
& =R(X, Y) Z-\frac{1}{n-1}[S(Y, Z) X-S(X, Z) Y],
\end{aligned}
$$

for any $X, Y, Z \in \chi(M)$ (Yano \& Kon, 1984). From (22), it follows that

$$
P(\xi, Y) Z=-g(Y, Z) \xi-\frac{1}{n-1} S(Y, Z) \xi .
$$

Now, we suppose that the manifold $M$ satisfies the condition $(P(\xi, X) \cdot B)(U, V) Z=0$. Then by definition we have

$$
\begin{aligned}
0= & P(\xi, X) B(U, V) Z-B(P(\xi, X) U, V) Z \\
& -B(U, P(\xi, X) V) Z-B(U, V) P(\xi, X) Z .
\end{aligned}
$$

Using (23) in (24) we obtain

$$
\begin{aligned}
& g(X, B(U, V) Z) \xi-g(X, U) B(\xi, V) Z \\
& -g(X, V) B(U, \xi) Z-g(X, Z) B(U, V) \xi \\
& +\frac{1}{n-1}[S(X, B(U, V) Z) \xi-S(X, U) B(\xi, V) Z \\
& -S(X, V) B(U, \xi) Z-S(X, Z) B(U, V) \xi]=0 .
\end{aligned}
$$

Using (14) in (25) we get

$$
\begin{aligned}
0= & g(X, B(U, V) Z) \xi \\
& +\frac{1}{n-1} S(X, B(U, V) Z) \xi .
\end{aligned}
$$

Taking inner product on both sides of (26) by $\xi$ we get

$$
\begin{aligned}
0= & (n-1) g(X, B(U, V) Z) \\
& +S(X, B(U, V) Z) .
\end{aligned}
$$

This implies that

$$
S(X, W)=-(n-1) g(X, W) .
$$

Thus the manifold is an Einstein manifold. Now, taking an orthonormal frame field and contracting over $X$ and $W$ in (28) we have

$$
r=-n(n-1,)
$$

where $r$ is the scalar curvature. In view of (28) and (29), the theorem is proved.

Theorem 3. If a Kenmotsu manifold $M^{n}$ satisfies the condition $\tilde{C}(\xi, X) \cdot B=0$, then either the scalar curvature is $r=-n(n-1)$ or the manifold is Dconformally flat.

Proof. Let $M$ be an $n$-dimensioal Kenmotsu manifold. The concircular curvature tensor $\tilde{C}$ of type $(1,3)$ on a Riemannian manifold $(M, g)$ of dimension $n$ is defined by

$$
\begin{aligned}
\tilde{C} & (X, Y) Z \\
= & R(X, Y) Z-\frac{r}{n(n-1)}[g(Y, Z) X \\
& -g(X, Z) Y]
\end{aligned}
$$

(Yano \& Kon, 1984). From (30), we have

$$
\begin{aligned}
& \tilde{C}(\xi, Y) Z \\
& =\left(1+\frac{r}{n(n-1)}\right)[\eta(Z) Y-g(Y, Z) \xi] .
\end{aligned}
$$

We suppose that the manifold $M$ satisfies the condition $(\tilde{C}(\xi, X) \cdot B)(U, V) Z=0$. Then we have

$$
\begin{aligned}
0= & \tilde{C}(\xi, X) B(U, V) Z-B(\tilde{C}(\xi, X) U, V) Z \\
& -B(U, \tilde{C}(\xi, X) V) Z-B(U, V) \tilde{C}(\xi, X) Z
\end{aligned}
$$

By virtue of (31) and (32), we obtain

$$
\begin{aligned}
& \left(1+\frac{r}{n(n-1)}\right)[\eta(B(U, V) Z) X \\
& -g(X, B(U, V) Z) \xi-\eta(U) B(X, V) Z \\
& +g(X, U) B(\xi, V) Z-\eta(V) B(U, X) Z \\
& +g(X, V) B(U, \xi) Z-\eta(Z) B(U, V) X \\
& +g(X, Z) B(U, V) \xi]=0 .
\end{aligned}
$$

By the use of (14) and (15) in (33), (33) reduces to

$$
\begin{aligned}
& \left(1+\frac{r}{n(n-1)}\right)[g(X, B(U, V) Z) \xi \\
& +\eta(U) B(X, V) Z+\eta(V) B(U, X) Z \\
& +\eta(Z) B(U, V) X]=0
\end{aligned}
$$

Taking inner product on both sides of (34) by $\xi$ and using (1) and (15), we get

$$
\left(1+\frac{r}{n(n-1)}\right) g(X, B(U, V) Z)=0 .
$$

This implies that either the scalar curvature is $r=-n(n-1)$ or $g(X, B(U, V) Z)=0$. 
From $g(X, B(U, V) Z)=0$, we have

$$
B(U, V) Z=0 .
$$

Hence the manifold is D-conformally flat. This completes the proof of the theorem.

Theorem 4. In an $n$-dimensional Kenmotsu manifold $M$ if the condition $C(\xi, X) \cdot B=0$ holds, then the manifold is an Einstein manifold with scalar curvature $r=-n(n-1)$.

Proof. Let us consider an $n$-dimensional Kenmotsu manifold $M$. The Weyl conformal curvature tensor $C$ of type $(1,3)$ on a Riemannian manifold $(M, g)$ of dimension $n$ is defined by

$C(X, Y) Z$

$=R(X, Y) Z-\frac{1}{n-2}[S(Y, Z) X-S(X, Z) Y$

$+g(Y, Z) Q X-g(X, Z) Q Y]$

$+\frac{r}{(n-1)(n-2)}[g(Y, Z) X-g(X, Z) Y]$

(Yano \& Kon, 1984). From (37), we have

$$
\begin{aligned}
& C(\xi, Y) Z \\
& =\frac{n+r-1}{(n-1)(n-2)}[g(Y, Z) \xi-\eta(Z) Y] \\
& -\frac{1}{n-2}[S(Y, Z) \xi-\eta(Z) Q Y] .
\end{aligned}
$$

Suppose that $M$ satisfies the condition $(C(\xi, X) \cdot B)(U, V) Z=0$. Then we have

$$
\begin{aligned}
0 & =C(\xi, X) B(U, V) Z-B(C(\xi, X) U, V) Z \\
& -B(U, C(\xi, X) V) Z-B(U, V) C(\xi, X) Z .
\end{aligned}
$$

By the use of (38) in (39), we obtain

$$
\begin{aligned}
& \left(\frac{n+r-1}{(n-1)(n-2)}\right)[g(X, B(U, V) Z) \xi \\
& -\eta(B(U, V) Z) X-g(X, U) B(\xi, V) Z \\
& +\eta(U) B(X, V) Z-g(X, V) B(U, \xi) Z \\
& +\eta(V) B(U, X) Z-g(X, Z) B(U, V) \xi \\
& +\eta(Z) B(U, V) X]-\frac{1}{n-2}[S(X, B(U, V) Z) \xi \\
& +\eta(B(U, V) Z) Q X-S(X, U) B(\xi, V) Z \\
& +\eta(U) B(Q X, V) Z-S(X, V) B(U, \xi) Z \\
& +\eta(V) B(U, Q X) Z-S(X, Z) B(U, V) \xi \\
& +\eta(Z) B(U, V) Q X]=0 .
\end{aligned}
$$

Using (14) and (15) in (40), we get

$$
\begin{aligned}
& \left(\frac{n+r-1}{(n-1)(n-2)}\right)[g(X, B(U, V) Z) \xi \\
& +\eta(U) B(X, V) Z+\eta(V) B(U, X) Z \\
& +\eta(Z) B(U, V) X]-\frac{1}{n-2}[S(X, B(U, V) Z) \xi \\
& +\eta(U) B(Q X, V) Z+\eta(V) B(U, Q X) Z \\
& +\eta(Z) B(U, V) Q X]=0 .
\end{aligned}
$$

Taking inner product on both sides of (41) by $\xi$ and using (1) and (15) we obtain

$$
\begin{aligned}
& \left(\frac{n+r-1}{(n-1)(n-2)}\right) g(X, B(U, V) Z) \\
& -\frac{1}{n-2} S(X, B(U, V) Z)=0 .
\end{aligned}
$$

From this equation it follows that

$$
S(X, W)=\frac{n+r-1}{n-1} g(X, W) .
$$

Taking an orthonormal frame field and contracting over $X$ and $W$ in (43), we get

$$
r=-n(n-1) \text {. }
$$

In view of (43) and (44), the theorem is proved.

Theorem 5. A Kenmotsu manifold $M^{n}$ satisfying the condition $S(X, \xi) \cdot B=0$ is D-conformally flat.

Proof. Consider an $n$-dimensional Kenmotsu manifold $M$ satisfying the condition

$$
S(X, \xi) \cdot B(U, V) Z=0 \text {. }
$$

By definition we have

$(S(X, \xi) \cdot B)(U, V) Z$

$=\left(\left(X \wedge_{s} \xi\right) \cdot B\right)(U, V) Z$

$=\left(X \wedge_{s} \xi\right) B(U, V) Z+B\left(\left(X \wedge_{s} \xi\right) U, V\right) Z$

$+B\left(U,\left(X \wedge_{s} \xi\right) V\right) Z+B(U, V)\left(X \wedge_{s} \xi\right) Z$,

where the endomorphism $X \wedge_{s} Y$ is defined as

$\left(X \wedge_{S} Y\right) Z=S(Y, Z) X-S(X, Z) Y$.

In view of (45), (46) and (47), we get

$$
\begin{aligned}
& 0=S(B(U, V) Z, \xi) X-S(X, B(U, V) Z) \xi \\
& +S(U, \xi) B(X, V) Z-S(X, U) B(\xi, V) Z \\
& +S(V, \xi) B(U, X) Z-S(X, V) B(U, \xi) Z \\
& +S(Z, \xi) B(U, V) X-S(X, Z) B(U, V) \xi .
\end{aligned}
$$

By the use of (10) and (14) in (48), we get 


$$
\begin{aligned}
& (n-1)[\eta(B(U, V) Z) X+\eta(U) B(X, V) Z \\
& +\eta(V) B(U, X) Z+\eta(Z) B(U, V) X] \\
& +S(X, B(U, V) Z) \xi .
\end{aligned}
$$

Taking inner product on both sides of (49) by $\xi$ and using (1), (3) and (15), we obtain

$$
S(X, B(U, V) Z)=0 \text {. }
$$

This equation implies that

$$
B(U, V) Z=0 \text {. }
$$

Thus the manifold is D-conformally flat. This completes the proof of the theorem.

Theorem 6. If a Kenmotsu manifold $M^{n}$ is $\varphi$-Dconformally flat, then the manifold is an Einstein manifold with scalar curvature $r=-n(n-1)$.

Proof. Let us consider an $n$-dimensional Kenmotsu manifold $M$ which is $\varphi$-D-conformally flat. Then the condition $g(B(\varphi X, \varphi Y) \varphi Z) \varphi W=0$ is satisfied. From (13), for $\varphi$-D-conformally flat it follows that

$$
\begin{aligned}
& g(R(\varphi X, \varphi Y) \varphi Z, \varphi W) \\
& +\frac{1}{n-3}[S(\varphi X, \varphi Z) g(\varphi Y, \varphi W) \\
& -S(\varphi Y, \varphi Z) g(\varphi X, \varphi W) \\
& +g(\varphi X, \varphi Z) S(\varphi Y, \varphi W) \\
& -S(\varphi X, \varphi W) g(\varphi Y, \varphi Z)] \\
& -\frac{K-2}{n-3}[g(\varphi X, \varphi Z) g(\varphi Y, \varphi W) \\
& -g(\varphi Y, \varphi Z) g(\varphi X, \varphi W)]=0 .
\end{aligned}
$$

Using (2), (7) and (12) in (52), we get

$$
\begin{aligned}
& \left(\frac{n-K-1}{n-3}\right)[\{g(X, Z)-\eta(X) \eta(Z)\} \\
& \times\{g(Y, W)-\eta(Y) \eta(W)\}-\{g(Y, Z) \\
& -\eta(Y) \eta(Z)\}\{g(X, W)-\eta(X) \eta(W)\}] \\
& +\frac{1}{n-3}[\{S(X, Z)+(n-1) \eta(X) \eta(Z)\} \\
& \times\{g(Y, W)-\eta(Y) \eta(W)\}-\{S(Y, Z) \\
& +(n-1) \eta(Y) \eta(Z)\}\{g(X, W)-\eta(X) \eta(W)\} \\
& +\{S(Y, W)+(n-1) \eta(Y) \eta(W)\}\{g(X, Z) \\
& -\eta(X) \eta(Z)\}-\{S(X, W)+(n-1) \eta(X) \eta(W)\} \\
& \times\{g(Y, Z)-\eta(Y) \eta(Z)\}]=0 .
\end{aligned}
$$

Let $\left\{e_{i}: i=1,2, \ldots, n\right\}$ be an orthonormal basis of the tangent space at any point of the manifold. Putting
$X=W=e_{i}$ in (53) and taking summation over $i, 1 \leq i \leq n$, we get

$$
\begin{aligned}
S(Y, Z)= & -\frac{n^{2}-K(n-2)+2 n+1+r}{n-3} g(Y, Z) \\
& +\frac{2(n-1)-K(n-2)+r}{n-3} \eta(Y) \eta(Z) .
\end{aligned}
$$

Putting $K=\frac{2(n-1)+r}{n-2}$ in (54) from (13), we obtain

$S(Y, Z)=-(n-1) g(Y, Z)$.

Thus the manifold is an Einstein manifold. Now, taking an orthonormal frame field and contracting over $Y$ and $Z$ in (55), we get

$r=-n(n-1)$.

By virtue of (55) and (56), the theorem is proved.

EXAMPLE OF A 3-DIMENSIONAL KENMOTSU MANIFOLD

We consider 3-dimensional manifold $M=\left\{(x, y, z) \in R^{3}\right\}, z \neq 0$ where $(x, y, z)$ are the standard coordinates of $R^{3}$.The vector fields

$e_{1}=z \frac{\partial}{\partial x}, e_{2}=z \frac{\partial}{\partial y}, e_{3}=-z \frac{\partial}{\partial z}$,

are linearly independent at each point of $M$. Let $g$ be the Riemannian metric defined by

$$
\begin{aligned}
& g\left(e_{1}, e_{2}\right)=g\left(e_{2}, e_{3}\right)=g\left(e_{1}, e_{3}\right)=0, \\
& g\left(e_{1}, e_{1}\right)=g\left(e_{2}, e_{2}\right)=g\left(e_{3}, e_{3}\right)=1 .
\end{aligned}
$$

Let $\eta$ be the 1 -form defined by $\eta(X)=g\left(X, e_{3}\right)$ for any $X \in \chi(M)$, the set of vector fields. Let $\varphi$ be $(1$, 1) tensor field defined by

$$
\varphi\left(e_{1}\right)=-e_{2}, \varphi\left(e_{2}\right)=e_{1}, \varphi\left(e_{3}\right)=0 .
$$

Then using the linearity of $\varphi$ and $g$, we have

$$
\begin{aligned}
& \eta\left(e_{3}\right)=1, \varphi^{2}(X)=-X+\eta(X) e_{3}, \\
& g(\varphi X, \varphi Y)=g(X, Y)-\eta(X) \eta(Y),
\end{aligned}
$$

for any vector fields $X, Y \in \chi(M)$. Thus for $e_{3}=\xi$, $(\varphi, \xi, \eta, g)$ defines an almost contact metric 
structure on $M$. Let $\nabla$ be the Levi-Civita connection with respect to the Riemannian metric $g$. Then by the definition of Lie bracket and (57), we have

$$
\begin{aligned}
{\left[e_{2}, e_{3}\right] } & =e_{2} e_{3}-e_{3} e_{2} \\
& =z \frac{\partial}{\partial y}\left(-z \frac{\partial}{\partial z}\right)-\left(-z \frac{\partial}{\partial z}\right)\left(z \frac{\partial}{\partial y}\right) \\
& =-z^{2} \frac{\partial^{2}}{\partial y \partial z}+z\left(z \frac{\partial^{2}}{\partial z \partial y}+\frac{\partial}{\partial y}\right) \\
& =z \frac{\partial}{\partial y} \\
& =e_{2} .
\end{aligned}
$$

Similarly, we obtain $\left[e_{1}, e_{2}\right]=0$ and $\left[e_{1}, e_{3}\right]=e_{1}$. Thus we have

$\left[e_{1}, e_{2}\right]=0,\left[e_{1}, e_{3}\right]=e_{1},\left[e_{2}, e_{3}\right]=e_{2}$.

The Levi-Civita connection $\nabla$ of the Riemannian metric $g$ is given by

$$
\begin{aligned}
& 2 g\left(\nabla_{X} Y, Z\right) \\
& =X g(Y, Z)+Y g(Z, X)-Z g(X, Y) \\
& +g([X, Y], Z)-g([Y, Z], X)+g([Z, X], Y),
\end{aligned}
$$

which is known as Koszul's formula.

By virtue of (58), (59), (63) and (64), we get

$$
\begin{aligned}
& 2 g\left(\nabla_{e_{1}} e_{3}, e_{1}\right) \\
& =e_{1} g\left(e_{3}, e_{1}\right)+e_{3} g\left(e_{1}, e_{1}\right)-e_{1} g\left(e_{1}, e_{3}\right) \\
& +g\left(\left[e_{1}, e_{3}\right], e_{1}\right)-g\left(\left[e_{3}, e_{1}\right], e_{1}\right)+g\left(\left[e_{1}, e_{1}\right], e_{3}\right) \\
& =2 g\left(e_{1}, e_{1}\right) .
\end{aligned}
$$

Similarly, we can calculate

$$
\begin{aligned}
& 2 g\left(\nabla_{e_{1}} e_{3}, e_{2}\right)=0=2 g\left(e_{1}, e_{2}\right) \text { and } 2 g\left(\nabla_{e_{1}} e_{3}, e_{3}\right)=0= \\
& 2 g\left(e_{1}, e_{3}\right) .
\end{aligned}
$$

Thus from above calculation we can write

$$
2 g\left(\nabla_{e_{1}} e_{3}, X\right)=2 g\left(e_{1}, X\right) \text {, }
$$

for all $X \in \chi(M)$. Hence we have $\nabla_{e_{1}} e_{3}=e_{1}$. Therefore, proceeding same way we obtain

$$
\left\{\begin{array}{l}
\nabla_{e_{1}} e_{3}=e_{1}, \nabla_{e_{1}} e_{2}=0, \nabla_{e_{1}} e_{1}=-e_{3}, \\
\nabla_{e_{2}} e_{3}=e_{2}, \nabla_{e_{3}} e_{2}=e_{3}, \nabla_{e_{2}} e_{1}=0, \\
\nabla_{e_{3}} e_{1}=\nabla_{e_{3}} e_{2}=\nabla_{e_{3}} e_{3}=0,
\end{array}\right.
$$

For $e_{3}=\xi$, (65) implies that

$$
\begin{aligned}
& \nabla_{e_{1}} e_{3}=e_{1}=e_{1}-g\left(e_{1}, e_{3}\right) e_{3}, \\
& \nabla_{e_{2}} e_{3}=e_{2}=e_{2}-g\left(e_{2}, e_{3}\right) e_{3}, \\
& \nabla_{e_{3}} e_{3}=0=e_{3}-g\left(e_{3}, e_{3}\right) e_{3},
\end{aligned}
$$

thus we have $\nabla_{X} \xi=X-g(X, \xi) \xi=X-\eta(X) \xi$, for $e_{3}=\xi$. Hence the manifold satisfies the condition (5).

Again, using (60) and (65) we obtain

$$
\begin{aligned}
\left(\nabla_{e_{1}} \varphi\right) e_{1}=\nabla_{e_{1}} \varphi e_{1}-\varphi \nabla_{e_{1}} e_{1} & =\nabla_{e_{1}}\left(-e_{2}\right)-\varphi\left(-e_{3}\right) \\
& =0 .
\end{aligned}
$$

Similarly, we can easily verify other relations and we have

$$
\left\{\begin{array}{l}
\left(\nabla_{e_{1}} \varphi\right) e_{1}=0,\left(\nabla_{e_{1}} \varphi\right) e_{2}=-e_{3},\left(\nabla_{e_{1}} \varphi\right) e_{3}=-e_{2}, \\
\left(\nabla_{e_{2}} \varphi\right) e_{1}=-e_{3},\left(\nabla_{e_{2}} \varphi\right) e_{2}=0,\left(\nabla_{e_{2}} \varphi\right) e_{3}=e_{1}, \\
\left(\nabla_{e_{3}} \varphi\right) e_{1}=\left(\nabla_{e_{3}} \varphi\right) e_{2}=\left(\nabla_{e_{3}} \varphi\right) e_{3}=0 .
\end{array}\right.
$$

From (4), we have $\left(\nabla_{X} \varphi\right) Y=g(\varphi X, Y) \xi-\eta(Y) \varphi X$. Using this relation with (58)-(60), we obtain

$$
\begin{aligned}
\left(\nabla_{e_{1}} \varphi\right) e_{1} & =g\left(\varphi e_{1}, e_{1}\right) e_{3}-\eta\left(e_{1}\right) \varphi e_{1} \\
& =g\left(-e_{2}, e_{1}\right) e_{3}-g\left(e_{1}, e_{3}\right)\left(-e_{2}\right) \\
& =0
\end{aligned}
$$

for $e_{3}=\xi$. Similarly, we can verify other relations and the manifold also satisfies the condition (4). From above it follows that the conditions (4) and (5) are satisfied by the manifold for $e_{3}=\xi$ and consequently the manifold under the consideration is a 3-dimensional Kenmotsu manifold.

\section{CONCLUSION}

In this paper, we have proved that an $n(n=2 m+1)$ dimensional Kenmotsu manifold satisfying curvature conditions $B(\xi, X) \cdot B=0, \quad \tilde{C}(\xi, X) \cdot B=0$ and $S(X, \xi) \cdot B=0$ is D-conformally flat. It also proved that Kenmotsu manifold satisfying the curvature conditions $P(\xi, X) \cdot B=0, C(\xi, X) \cdot B=0$ and $g(B(\varphi X, \varphi Y) \varphi Z, \varphi W)=0$ is an Einstein manifold with scalar curvature $r=-n(n-1)$.

The paper will be useful for those who are working and studying in the field of structures on differentiable manifolds. 


\section{ACKNOWLEDGEMENT}

The author is grateful to the worthy referee for his/her useful suggestions for the improvement of the paper.

\section{REFERENCES}

Blair, D. E. (1976). Contact manifolds in Riemannian geometry. Lecture Notes in Mathematics. Springer-Verlag, Berlin, 509.

Blair, D. E. (2002). Riemannian geometry of contact and sympletic manifolds. Progress Mathematics. Birkhauser, Boston, 203.

Chuman, G. (1983). On the D-conformal curvature tensor. Tensor N. S., 46: 125-129.

De, U. C. (2008). On $\varphi$-symmetric Kenmotsu manifolds. International Electronic Journal of Geometry, 1 (1): 33-38.
De, U. C. and Pathak, G. (2004). On 3-dimensional Kenmotsu manifolds. Indian Journal of Pure Applied Mathematics, 35: 159-165.

De, U. C.; Yildiz, A. and Yaliniz, A. F. (2009). On $\varphi$-recurrent Kenmotsu manifolds. Turk Journal of Mathematics, 33: 17-25.

Jun, J. B.; De, U. C. and Pathak, G. (2005). On Kenmotsu manifolds. Journal of Korean Mathematics Society, 42: 435-445.

Kenmotsu, K. (1972). A class of almost contact Riemannian manifolds. Tohoku Mathematics Journal, 24: 93-103.

Yano, K. and Kon, M. (1984). Structures on manifolds. Series in Pure Mathematics. World Scientific Pub. Co. Pvt. Ltd., Singapore. 